\title{
Multistage Latissimus Dorsi Flap with Implant for Complex Post-Mastectomy Reconstruction: An Old but Still Current Technique
}

\author{
Francesca De Lorenzi ${ }^{a}$ Francesco Borelli ${ }^{a}$ Pietro Sala ${ }^{a}$ Pietro Loschi ${ }^{a}$ \\ Paola Naninato $^{b} \quad$ Anna Rita Vento $^{b}$ Elisabetta Maria Cristina Rossi ${ }^{b}$ \\ Paolo Veronesi ${ }^{\text {b }}$ \\ ${ }^{\mathrm{a} D i v i s i o n}$ of Plastic and Reconstructive Surgery, European Institute of Oncology, Milan, Italy; ${ }^{\mathrm{b}}$ Division of Breast \\ Surgery, University of Milan, European Institute of Oncology, Milan, Italy
}

\section{Keywords}

Latissimus dorsi flap · Implant · Breast reconstruction

\begin{abstract}
Introduction: The latissimus dorsi (LD) flap has been used for reconstructing mastectomy defects since the early 1900 s. Although its popularity has declined over the last decades, it still retains an important role in breast reconstruction. We present our recent experience with the multistage LD flap and implant for extremely complex post-mastectomy defects. Patients and Methods: Between 2011 and 2020, 42 consecutive patients underwent post-mastectomy LD reconstruction with an expander (STAGE 1). Some of them received prior fat-grafting of the mammary region (STAGE 0). All patients were scheduled for an expander-definitive implant change (STAGE 2). Some of them completed the program with fat-grafting, nipple and areola reconstruction, and other refinements (STAGE 3 or 4). Results: Two patients underwent fat-grafting at STAGE 0. Mean age at STAGE 1 was 46.7 years, mean BMI was $23.6,14.4 \%$ of the patients were smokers, and $21.4 \%$ had comorbidities. Immediate reconstructions were performed in $35.7 \%$ and delayed in $64.3 \%$. Mean surgical time at STAGE 1 was 194.7 min for delayed reconstructions and $242.3 \mathrm{~min}$ for immediate ones. Mean hospital stay for STAGE 1 procedures was 3.8 days; all other STAGES were performed as ambulatory surgery. No flap necrosis was observed and only 1 patient required a surgical
\end{abstract}

revision for bleeding. Dorsal seroma occurred in $45.2 \%$ of cases. Conclusions: The multistage LD flap with implant is a useful and safe tool within the reconstructive armamentarium for post-mastectomy defects. It combines multiple simple procedures and does not require specific skills and surgical training (level of evidence 4).

(c) 2020 S. Karger AG, Basel

\section{Introduction}

The latissimus dorsi (LD) flap has been used for reconstructing mastectomy defects for more than 100 years. First described by Iginio Tansini in the early 1900s [1], its anatomy and the harvesting technique were detailed by Olivari [2] in 1976. Its consistent vascular anatomy and a robust and long pedicle as well as a quick postoperative recovery made the $\mathrm{LD}$ flap an attractive tool for breast reconstruction. Several refinements [3-5] and indications [6] have been described over the years. It has been used to correct partial breast defects after conservation, in both the immediate setting (oncoplastic replacement procedures) [7] and for breast asymmetries in the delayed setting. It can be used for total breast reconstruction with or without implants (autologous LD flap) [8], or as a salvage procedure for secondary reconstructions (implant or free-flap failure). Finally, it has been used to repair chest wall defects in the V-Y fashion [9]. Despite its long 
Table 1. Patients' characteristics at STAGE 1

\begin{tabular}{lc}
\hline Variable & Total $(n=42)$ \\
\hline Age, years & $40.9(31-75)$ \\
Smoker & \\
Yes & $12(14.4)$ \\
No & $30(71.4)$ \\
BMI & $23.61(17.9-36.9)$ \\
Comorbidities & \\
Yes & $9(21.4)$ \\
No & $33(78.6)$ \\
Histology & \\
DCIS & $4(9.5)$ \\
IDC & $28(66.7)$ \\
ILC & $8(19)$ \\
Unknown & $2(4.8)$ \\
pT & \\
Tis & $3(7.1)$ \\
pT1 & $7(16.6)$ \\
pT2 & $12(14.4)$ \\
pT3 & $7(16.6)$ \\
pT4 & $5(11.9)$ \\
X & $3(7.1)$ \\
Radiotherapy & \\
Yes & $42(100)$ \\
No & 0 \\
Chemotherapy & \\
Adjuvant & $33(78.5)$ \\
Neoadjuvant & $17(40.4)$ \\
No & $2(4.8)$ \\
Hormonal therapy & $38(90.5)$ \\
Yes & $4(9.5)$ \\
No & \\
\hline & \\
\hline &
\end{tabular}

Values are expressed as $n$ (\%) or median (range). pT is referred at the time of the mastectomy.

history and established strengths, the LD flap has declined in popularity as the first choice for reconstruction over the last decades. It is now the time of conservative mastectomies and anatomically shaped form-stable implants, perforator [10] and free flaps, fat-grafting, biological matrices, and synthetic meshes.

Despite recent trends, the LD flap still plays an important role in breast reconstruction. According to the National American Impatient Sample Dataset, 2,304 LD flaps were performed for post-mastectomy reconstruction between 2008 and 2010 [11].

We present our recent experience with the multistage LD flap and implant for extremely complex post-mastectomy defects.

\section{Materials and Methods}

Between 2011 and 2020, 42 consecutive patients underwent post-mastectomy $\mathrm{LD}$ flap reconstruction with an expander (STAGE 1) in the immediate or delayed setting. All patients were operated on by the senior author (F.D.L.). They represent all LD flap reconstructions performed during this period, except for those after conservative surgery, chest wall defects after thoracic, melanoma, or sarcoma surgery.

Flap harvesting was performed with the patient positioned in the lateral decubitus. In the delayed procedures, the mastectomy scar was incised. At the site of the mastectomy, the anterior border of the LD and the thoracodorsal pedicle were identified. The skin paddle was incised, and the superficial muscular face and deep face were detached. The muscle was resected and rotated toward the mammary region. We routinely partially detach the tendon of LD from its insertion to allow the advancement of the flap towards the mammary region without tension and avoid bulging in the axilla. We never transect the thoracodorsal nerve.

The dimensions and shapes of the dorsal skin paddle vary a lot according to different mammary defects, which requires tailoring of the flap remodeling for each patient. The implant is placed behind the flap above the thoracic cage.

The site was closed in a multilayer fashion with absorbable stitches without using Baroudi's sutures or fibrin glue. All patients received preoperative irradiation. Patients' characteristics at STAGE 1 are reported in Table 1. Some patients received prior fatgrafting of the mammary region to improve the tissue quality and facilitate scar release if needed (STAGE 0). This procedure was performed as ambulatory surgery.

All patients were scheduled for expander-definitive implant change in ambulatory surgery (STAGE 2). Simultaneous contralateral mammaplasty was performed if required. Some of them completed the reconstruction with fat-grafting, nipple and areola reconstruction, and other refinements (STAGE 3 or 4). A flowchart of the patients and the multistaged reconstruction appears in Figure 1.

Patient and surgeon satisfaction were scored on a scale of $0-10$. Statistical analysis was performed using IBM SPSS software v24.0. A descriptive analysis was carried out of patients' history and characteristics at the time of primary surgery; data are presented as frequencies and percentages.

\section{Results}

Three patients (7.2\%) underwent fat-grafting in the mammary region (STAGE 0) before LD flap and expander reconstruction.

Mean age at STAGE 1 was 46.7 years (range $31-75$ ), mean BMI was 23.6 (range 17.9-36.7), 14.4\% patients were smokers, and $21.4 \%$ had at least 1 comorbidity (hypertension, diabetes, atrial fibrillation, or endocrine disorders).

The LD flap and expander reconstruction was performed after implant exposure and infection in 11 patients $(26.1 \%)$, after a skin burn in $1(2.4 \%)$, after salvage mastectomy for local relapses in $15(35.7 \%)$, and for complex breast defects in 15 (35.7\%).

Immediate reconstructions were performed in 35.7\% and the remaining $64.3 \%$ were delayed procedures.

All post-mastectomy LD flap and expander reconstructions were monolateral, although 3 patients underwent bilateral mastectomy and reconstruction at STAGE 1. 


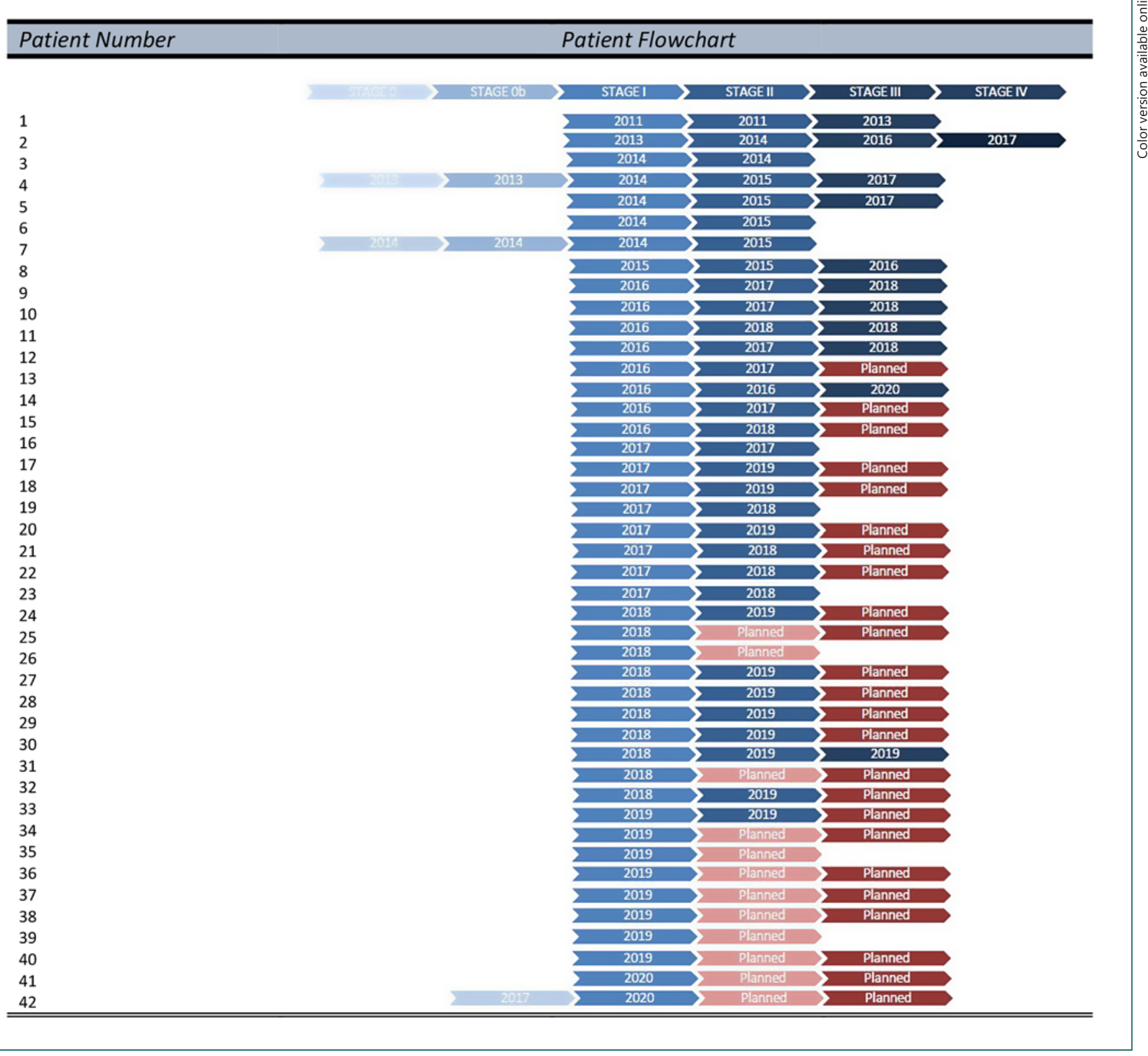

Fig. 1. Patient flowchart and multistaged reconstruction.

Mean duration of surgery at STAGE 1 was $194.7 \mathrm{~min}$ (range 156-276) for delayed reconstructions and 242.3 min (range 193-294) including mastectomy and axillary surgery if planned.

Mean hospital stay for STAGE 1 procedures was 3.8 days (range 2-8). All other stages were performed as ambulatory surgery, except for in 5 patients who required contralateral mastectomy and reconstruction.

No flap necrosis (partial or complete) was observed in the series. Skin paddle shape and dimensions largely varied from extremely small, corresponding to half the size of the areola, to large, corresponding to all superior quadrants (Fig. 2), or even more according to oncologic resec- tion of the remaining local tissue. The mean volume of the tissue expander was $427.5 \mathrm{~mL}$ for STAGE 1 (range 300-600). One patient required surgical revision for bleeding (2.4\%). No sensate flap procedures were performed in the series.

Dorsal seroma occurred in $45.2 \%$ of cases. Breast seroma occurred in 1 patient (2.4\%). No other complications, e.g., the loss, exposure, or dislocation of the implant, postsurgical neuroma, wound dehiscence, infection, or skin necrosis were recorded. We did not evaluate capsular contracture due to relative short follow-up period.

Thirty patients $(71.4 \%)$ completed STAGE 2 . The mean volume of the definitive implant was $424 \mathrm{~mL}$ (range 


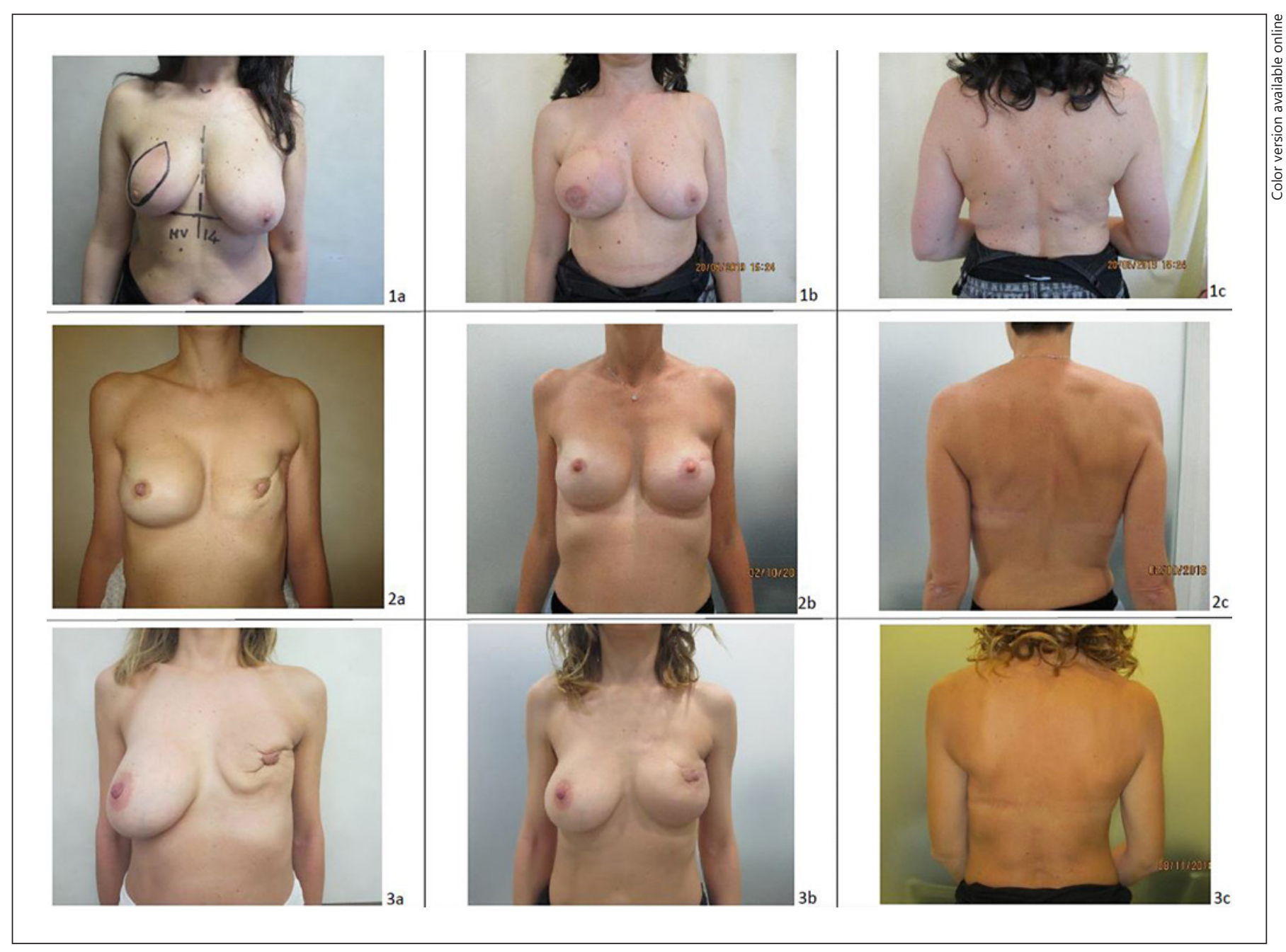

Fig. 2. 1a: A 45-year-old woman presented with a 4-cm local relapse in her right breast after conservation. Right total mastectomy and LD and expander were planned. 1b: Final result after STAGE 3 , after nipple and areola reconstruction and fat-grafting in the upper pole and internal quadrants. 1c: Postoperative view of the dorsal region. 2a: A 40-year-old woman after a bilateral nipple-sparing mastectomy and definitive implant. In the left breast, infection oc- curred after irradiation, requiring removal of the implant. 2b: Final result after STAGE 2, the same-sized implant was placed in the left breast. 2c: Postoperative view of the dorsal region. 3a: A 39-yearold woman after implant failure in the left breast after irradiation. 3b: Final result after STAGE 2. The patient is waiting for left fatgrafting and nipple and areola repositioning. 3c: Postoperative view of the dorsal region.
295-625). At this time, contralateral mammaplasty was performed in $40.4 \%$ of patients including reduction mammaplasty (16.6\%), mastopexy (19\%), and augmentation mammoplasty (4.8\%). Five patients (11.9\%) underwent contralateral mastectomy and reconstruction at STAGE 2; 1 was a risk-reducing mastectomy and 4 were nipple areola-sparing mastectomies for new cancers. These patients underwent standard hospitalization. No surgery was performed in the contralateral breast in $40.4 \%$ of cases. In 1 patient (2.4\%), rotation of the implant occurred, requiring further surgery.

Fifteen patients completed the multistage reconstruction $(35.7 \%)$, with an average number of 3 procedures. Four underwent a two-step reconstruction, 8 had a 3-step reconstruction, 4 others required 4 steps, and 1 required 5 procedures. Good cosmetic results were achieved in all cases and the patients were satisfied.

Of the patients (64.3\%) who did not complete the multistage reconstruction, a definitive implant-expander change is planned for 12 of them, and 8 of these 12 will probably undergo nipple and areola reconstruction. Twelve patients are planned for nipple areola reconstruction under local anesthesia. Three patients require other refinements, including implant change, fat-grafting, and scar revision. Median follow-up is 21 months (range $1-80)$. 


\section{Discussion}

This study demonstrates that multistage LD flap with an implant is a valid alternative for complex post-mastectomy reconstruction, an old but still current technique. Our experience has proved it to be a safe and simple procedure able to address and manage extremely complex defects in irradiated breasts, particularly in salvage situations or after implant extrusion. It has excellent cosmetic outcomes. In our patients, we thought that only microsurgical transfers would achieve our goal. Sometimes women are not candidates for total autologous tissue transfer. The multistage LD flap has the great advantage of not requiring specific skills or training in microsurgery, and it can thus be implemented in every breast unit.

Although implant-based reconstruction is currently the most frequent procedure after mastectomy in our practice, the multistage LD flap is an important tool for extremely complex breast defects. Over the years, we have observed an evolution of oncologic surgical techniques, diagnostic tools, and implants. Less aggressive mastectomies are performed nowadays, thanks to oncological screening, allowing immediate reconstruction with definitive implants. We have abandoned $\mathrm{LD}$ reconstruction in cases of partial breast defects, asymmetries, and oncoplastic procedures. Fat-grafting has replaced LD reconstruction in the delayed setting and perforator flaps are used in the immediate setting. Nevertheless, LD reconstruction is still useful for salvage mastectomies and implant failure.

The key point of our technique is the combination of different simple procedures in a multistage fashion. This is an advantage, and, in our opinion, it should be added to the existing range of reconstructive approaches.

Fat-grafting in the native mastectomy flap (STAGE 0) before LD harvesting is reserved for more complex defects characterized by soft-tissue adhesion to the underlying bone. It facilitates further undermining of the mammary skin and allows its preservation for reconstruction. It is performed as ambulatory surgery. Fat-grafting is also used as a final refinement, usually associated with nipple and areola reconstruction (STAGES 3 and 4). In these cases, the upper pole is more frequently grafted to improve the superior contour and hidden implant border. None of the patients included in our series received fatgrafting in the skin paddle or in the muscle at the same time of $\mathrm{LD}$ reconstruction [12].

In our experience, even tissue expander change for definitive implant can be performed as ambulatory surgery. It is an expeditious operation and was associated with contralateral mammaplasty in $40.4 \%$ of patients in this series. The only stage that requires hospitalization is LD reconstruction with expander, or if the mastectomy and the reconstruction are performed simultaneously. The average stay in hospital for STAGE 1 is 3.8 days and the average operative time for this procedure is approximately $195 \mathrm{~min}$ in the delayed setting and $240 \mathrm{~min}$ in the immediate setting.

In our experience, the multistage LD and implant procedure is safe, with a low percentage of complications when compared to other series in the literature [5]. No flap necrosis or mastectomy flap necrosis was observed in the series. There was no scar dehiscence in the donor or recipient areas. Expander-implant LD reconstruction allows the removal of damaged tissue and non-viable mastectomy flaps. There is no tension of the mammary wound as the expander is deflated, and there is no tension in the dorsal area because no large skin paddle is required. We believe that all these factors positively influence low rate of complications.

In addition to the simplicity of the procedure, our experience highlights its versatility and the capacity to adapt to different and complex breast defects. The combination fat- grafting/expansion phenomenon improves the quality and quantity of the local tissues, making breast reconstruction possible and achieving optimal outcomes.

We fully agree with Feng et al. [13] that the two-stage LD flap with implant reconstruction allows the size of the definitive implant to be estimated after complete healing of the implant pocket and LD atrophy after systemic treatments that can interfere with a patient's body weight. However, this is not our reason for recommending multistage LD. In our belief, suitable candidates for the procedure are those women with complex breast defects and poor availability of local tissues, i.e., that do not allow the direct use of definitive implants in combination with LD or other one-stage reconstructions.

Patient satisfaction, LD morbidity, and the oncological safety of the procedure were not addressed in this paper since we focused on these issues in previous studies $[6,14$, 15]. Capsular contraction was also not evaluated due to the short follow-up period and can thus be considered a limitation of this study.

The procedure does have some limitations. A longstanding critique of the LD flap has been the widened and highly visible donor site scar. Adjustment of the skin island design can improve donor-site scar appearance. In our practice, orienting the skin island horizontally at the level of the bra minimizes scar visibility. It allows the patient to wear a bra without the scar being visible. Moreover, the multistage procedure reduces the need for a large dorsal skin paddle or extended LD, confining the scar behind the bra and decreasing donor-site morbidity.

The approach is time-consuming, requiring multiple procedures. However, in our experience, patients were well-informed about the complications associated with irradiated tissue and the poor availability of local tissue, and they were accepting of the multiple simple and safe procedures to achieve the desirable results. In our series, 
there was a long interval between each stage. This was due to the oncologic treatments for immediate STAGE 1 procedures, patient willingness, and, unfortunately, the waiting list for reconstructive procedures.

In conclusion, we do believe that the multistage $\mathrm{LD}$ flap with implant is a useful tool within the reconstructive armamentarium for post-mastectomy defects. It combines multiple simple procedures and does not require specific skills and surgical training. It is easily implemented in every breast unit. It is safe and allows for excellent cosmetic outcomes.

\section{Statement of Ethics}

The study protocol was approved by the European Institute of Oncology's Scientific Committee and Risk Management Decisional Unit. Written informed consent was obtained from all study participants. All patients mentioned in this paper signed a written consent to have their pictures/photos used for scientific purposes.

\section{Conflict of Interest Statement}

The authors have no conflicts of interest to declare.

\section{Funding Sources}

None of the authors received any funding for this work.

\section{Author Contributions}

All authors materially participated in this research, were involved in the preparation of this article, and approved the final version. F.D.L., F.B., and P.S. were involved in the study conception and design. P.L., P.N., A.R.V., P.V., and E.M.C.R. were involved in acquisition, analysis and interpretation of the data. F.D.L. and F.B. wrote the initial draft of this paper. P.S., P.L., P.N, A.R.V., E.M.C.R., and P.V. were involved in the critical revision of this work.

\section{References}

1 Maxwell GP. Iginio Tansini and the origin of the latissimus dorsi musculocutaneous flap. Plast Reconstr Surg. 1980 May;65(5):686-92.

2 Olivari N. The latissimus flap. Br J Plast Surg. 1976 Apr;29(2):126-8.

3 Maselli AM, Mella JR, Guo L. Refining the latissimus dorsi flap. Ann Plast Surg. 2019 Oct; 83(4S Suppl 1):S11-6.

4 de Runz A, Boccara D, Bekara F, Chaouat M, Mimoun M. Outcome of 122 delayed breast reconstruction following post-mastectomy radiotherapy: the scarless latissimus dorsi flap with tissue expansion technique. Ann Chir Plast Esthet. 2017 Feb;62(1):23-30.

5 Cook J, Waughtel J, Brooks C, Hardin D, Hwee YK, Barnavon Y. The muscle-sparing latissimus dorsi flap for breast reconstruction. A retrospective review of 126 consecutive flaps. Ann Plast Surg. 2017 Jun;78(6S Suppl 5):S263-8.

6 De Lorenzi F, Corso G, Botta F, Invento A, Marchetti A, Sala P, et al. Immediate breast reconstruction with latissimus dorsi flap for patients with local recurrence of breast cancer. Eur J Surg Oncol. 2020 Jun;46(6):1013-20.
7 Alço G, Igdem S, Okkan S, Dincer M, Sarsenov D, Ilgun AS, et al. Replacement of the tumor bed following oncoplastic breast-conserving surgery with immediate latissimus dorsi mini-flap. Mol Clin Oncol. 2016 Oct;5(4): 365-71.

8 Delay E, Florzac AS, Frobert P. Breast reconstruction with the autologous latissimus dorsi flap. Ann Chir Plast Esthet. 2018 Nov;63(5-6): 422-36. French.

9 Christen T, Koch N, Philandrianos C, Ramirez R, Raffoul W, Beldi M, et al. The V-Y latissimus dorsi musculocutaneous flap in the reconstruction of large posterior chest wall defects. Aesthetic Plast Surg. 2012 Jun;36(3): 618-22.

10 Hamdi M, Van Landuyt K, Monstrey S, Blondeel P. Pedicled perforator flaps in breast reconstruction: a new concept. Br J Plast Surg. 2004 Sep;57(6):531-9.

11 DeLong MR, Tandon VJ, Rudkin GH, Da Lio AL. Latissimus dorsi flap breast reconstruction - A nationwide inpatient sample review. Ann Plast Surg. 2017 May;78(5 Suppl 4):S185-8.
12 Brondi RS, de Oliveira VM, Bagnoli F, Mateus EF, Rinaldi JF. Autologous breast reconstruction with the latissimus dorsi muscle with immediate fat grafting: long-term results and patient satisfaction. Ann Plast Surg. 2019 Feb; 82(2):152-7.

13 Feng J, Pardoe CI, Mota AM, Chui CH, Tan BK. Two-stage latissimus dorsi flap with implant for unilateral breast reconstruction: getting the size right. Arch Plast Surg. 2016 Mar; 43(2):197-203.

14 Garusi C, Lohsiriwat V, Brenelli F, Galimberti VE, De Lorenzi F, Rietjens M, et al. The value of latissimus dorsi flap with implant reconstruction for total mastectomy after conservative breast cancer surgery recurrence. Breast. 2011 Apr;20(2):141-4.

15 Garusi C, Manconi A, Lanni G, Lomeo G, Loschi P, Simoncini MC, et al. Shoulder function after breast reconstruction with the latissimus dorsi flap: A prospective cohort study - Combining DASH score and objective evaluation. Breast. 2016 Jun;27:78-86. 